\title{
Comparison between self-gripping mesh and sutured mesh in open inguinal hernia repair
}

\author{
Mahmoud Mohamed Abou El-Yazid, Mahmoud Mohammed Mahran and Alaa Abdel Aty \\ Mahmoud \\ Department of General Surgery, Faculty of Medicine, Al-Azhar University \\ Corresponding author: Alaa Abdel Aty Mahmoud, Phone: (020) 01273999797 E-Mail: alaaabes2@ gmail.com
}

\begin{abstract}
Background: Inguinal hernias are one of the most common problems encountered by the surgeon, accounting for about $10-12 \%$ of all operations. Operations for the inguinal hernia are one of the most common procedures. Currently herniorrhaphy is the most performed surgical procedure in the world.

Aim: The aim of this study is to compare tissue adhesives and how it can reduce postoperative complications, especially chronic pain as well as recurrence rate in self-gripping mesh compared with sutured mesh in open inguinal hernia repair.

Methods: This study conducted on 30 cases of fresh inguinal hernia submitted to inguinal hernioplasty "mesh repair" 15 of them submitted to sutureless mesh "Group I" while the other 15 cases submitted to sutured mesh "Group II".

Results: Our results revealed that there was no statistical difference between both groups regarding the age. Also, there was no significant difference between both groups regarding gender. Our study revealed that the operative time was longer and blood loss was more during operation in sutured group than in sutureless group. In spite of increase incidence of complications sutured group than in sutureless in our study but there was no difference between both groups regarding complications.
\end{abstract}

Conclusion: From our study we can conclude that the use of self-grip (sutureless) meshes and sutured meshes in the management of groin hernias have nearly the same results in experienced hands.

Keywords: Self-gripping mesh, Sutured mesh, Inguinal hernia

\section{Introduction}

Inguinal hernias are one of the most common problems encountered by the surgeon, accounting for about $10-12 \%$ of all operations. An inguinal hernia can be defined as protrusion of a part or whole abdominal viscous into the inguinal canal either through the deep ring or through Hasselbach's triangle. Approximately $75 \%$ of all hernias occur in the groin with a lifetime risk of $27 \%$ in men and $3 \%$ in women. About 2/3 of these hernias are indirect and one third is direct ${ }^{(\mathbf{1})}$.

Operation for the inguinal hernia is one of the most common procedure that a general surgeon undertakes and various surgeons have given their valuable contribution to different techniques of inguinal hernia repair for better outcome in relation to various complications like groin pain, infection, testicular atrophy and recurrence etc. related to older techniques ${ }^{(2)}$.

Nowadays mesh hernia repair has become gold standard and increasing mesh procedure in hernia surgery led to substantial decrease in hernia recurrence rate ${ }^{(\mathbf{1})}$. The use of mesh has reduced recurrence rates to below $5 \%$ in inguinal hernia repair and post-operative morbidity is now often attributed to other outcome measures, especially chronic pain and surgical site infection (SSI) ${ }^{(3)}$.

The current surgical options for mesh fixation include, but are not limited to, sutures, tacks or staples, self-fixing meshes and fibrin or other glues. However, there is no consensus among doctors on the best surgical technique. The choice of options often depends on surgeons' personal preference ${ }^{(4)}$.

Heavy weight polypropylene meshes have been found to elicit inflammatory reactions responsible for mesh shrinkage when scar tissue evolves. Thus, it has been recommended to use low-weight meshes and to limit the extent of fixation. More recently, self-gripping meshes have been developed, avoiding the need for additional fixation. The Parietex ProGri selffixating mesh is composed of monofilament polyester and polylactic acid (PLA) grips, and is indicated for use in inguinal and incisional hernia repairs ${ }^{(5)}$. 
Authors advocating sutureless mesh hernioplasty, have the opinion that they decrease tension in suture line and a better leveling leads to rapid embodiment of mesh without formation of dead space therefore chances of nerve entrapment and post operative complications are reduced, so that postoperative recovery and postoperative hospital stay will be decreased. On the other hand some studies claim that chances of displacement, migration and folding of mesh are more in sutureless mesh hernioplasty than traditional Lichtenstein technique, resulting in the failure of the whole procedure ${ }^{(\mathbf{1})}$.

The sutureless ProGrip mesh is a revolutionary mesh as it can be secured without a suture, avoiding any risk for nerve entrapment, and preserving anatomical structures. Moreover, the resorbable PLA micro-grips of the ProGrip mesh are substantially blunt to prevent damage to the surrounding tissues ${ }^{(5)}$.

\section{Aim of the work}

The aim of this study is to compare tissue adhesives and how it can reduce postoperative complications, especially chronic pain as well as recurrence rate in self-gripping mesh compared with sutured mesh in open inguinal hernia repair.

\section{Patients and Methods}

This study was conducted at Al-Azhar Main University Hospitals and Kafr El-Dawar General Hospital on patients with inguinal hernias. The study was approved by the Ethics Board of Al-Azhar University.

This study was conducted in Surgical Departments on 30 patients with inguinal hernia classified into 2 groups:

Group I: $\quad 15$ patients underwent hernia repair with self-gripping "Prolene" mesh.

Group II: 15 patients underwent hernia repair with sutured "Prolene" mesh.

\section{Inclusion criteria:}

Patients with fresh inguinal hernia were included in our study.

\section{Exclusion criteria:}

- Patients with recurrent hernia

- Patients with severe liver affection

- Patients with permanent causes of increased intra-abdominal pressure

\section{Methods}

All studied patients were submitted to the following:

- History taking

- Presence of comorbidity that persist after repair as $\mathrm{BPH}$

- Clinical examination and laboratory investigations

- Operative assessment:

- Duration of surgery (operative time)

- Intraoperative difficulties

- Duration of postoperative stay and results

- Postoperative complications as infection, rejection of mesh, postoperative pain, etc..,

- Recurrence rate

- Follow-up of patients monthly for 6 months

\section{Statistical analysis}

Data were be collected and entered to the computer using SPSS (Statistical Package for Social Science) program for statistical analysis.

Two types of statistics were done:

\section{A. Descriptive statistics:}

* Quantitative data were shown as mean, $\mathrm{SD}$, and range.

* Qualitative data were expressed as frequency and percent.

\section{B. Analytical statistics:}

* Chi-square test was used to measure association between qualitative variables.

* $\quad$ Student t-test was used to compare means of 2 sets of quantitative normally distributed data, while Mann Whitney test was used when these data were not normally distributed.

* P-value is considered statistically significant when it is $\leq 0.05$.

\section{Results}

This study conducted on 30 cases of fresh inguinal hernia submitted to inguinal 
hernioplasty "mesh repair" 15 of them submitted to sutureless mesh "Group I" while the other 15 cases submitted to sutured mesh "Group II".

The age of group I ranged between 2570 years with a mean age of $55.9 \pm 14.4$ years while in group II the age of patients ranged between $30-68$ years with a mean of $57 \pm 10.7$ years. The statistical analysis revealed that there was no statistical significant difference between both groups regarding the age $(\mathrm{P}=$ $0.811)$.

All patients of group I $(15 / 15,100 \%)$ without any female $(0 / 15,0.0 \%)$ while in group II $93.3 \%(14 / 15)$ of patients were males and $6.7 \%(1 / 15)$ was female and the statistical analysis revealed that there was no significant difference between both groups regarding gender $(\mathrm{P}=0.845)$.

Table 1: Age and sex distribution in groups of the study.

\begin{tabular}{|c|c|c|c|c|c|}
\hline Variable & \multicolumn{2}{|c|}{ Group I } & \multicolumn{2}{|c|}{ Group II } & $\mathbf{P}$ \\
\hline $\begin{array}{l}\text { Age (Years): } \\
\text { Range } \\
\text { Mean } \pm \text { S.D }\end{array}$ & \multicolumn{2}{|c|}{$\begin{array}{c}25-70 \\
55.9 \pm 14.4\end{array}$} & \multicolumn{2}{|c|}{$\begin{array}{c}30-68 \\
57 \pm 10.7\end{array}$} & $\begin{array}{c}0.811 \\
(\mathrm{NS})\end{array}$ \\
\hline Sex & No & $\%$ & No & $\%$ & \\
\hline $\begin{array}{l}\text { Male } \\
\text { Female }\end{array}$ & $\begin{array}{c}15 \\
0\end{array}$ & $\begin{array}{c}100 \\
0\end{array}$ & $\begin{array}{c}14 \\
1\end{array}$ & $\begin{array}{c}93.3 \\
6.7\end{array}$ & $\begin{array}{c}0.845 \\
(\mathrm{NS})\end{array}$ \\
\hline Total & 15 & 100 & 15 & 100 & \\
\hline
\end{tabular}

In group I seven patients $(7 / 15,46.7 \%)$ had right indirect inguinal hernia (I.I.H), five patients $(5 / 15$, $33.3 \%)$ had left indirect inguinal hernia and three patients $(3 / 15,20 \%)$ had bilateral indirect inguinal hernia. While in group II eight patients $(8 / 15,53.3 \%)$ had right indirect inguinal hernia and seven patients $(7 / 15,46.7 \%)$ had left I.I.H. and the statistical analysis revealed that there was no significant difference between both groups regarding type of hernia $(\mathrm{P}=0.631)($ Table 2$)$.

Table 2: Age and sex distribution in groups of the study.

\begin{tabular}{|l|c|c|c|c|c|}
\hline \multirow{2}{*}{ Type of hernia } & \multicolumn{2}{|c|}{ Group I } & \multicolumn{2}{c|}{ Group II } & \multirow{2}{*}{ P } \\
\cline { 2 - 6 } & No & $\%$ & No & $\%$ & \\
\hline Right I. I. H & 7 & $46.7 \%$ & 8 & $53.3 \%$ & \multirow{2}{*}{$\mathbf{0 . 6 3 1}$} \\
Left I. I. H & 5 & $33.3 \%$ & 7 & $46.7 \%$ & (NS) \\
Bilateral I. I. H & 3 & $20 \%$ & 0 & $0.0 \%$ & \\
\hline Total & 15 & 100 & 15 & 100 & \\
\hline
\end{tabular}

All patients $(15 / 15,100 \%)$ of both groups underwent hernioplasty of anterior approach using mesh "synthetic polypropylene", without any intraoperative complications. In fifteen of the patients "Group I" the mesh put without sutures while the other 15 "Group II" the mesh was sutured.

The operative time in group I ranged between $40-58 \mathrm{~min}$ with a mean operative time of $50.3 \pm 4.7$ min while in group II the operative time ranged between 49-70 min with a mean operative time of $65.5 \pm 5.5 \mathrm{~min}$ and the statistical analysis revealed that there was significant statistical increase in the operative time of group II "sutured group" than in group I "sutureless group" ( $\mathrm{P}=0.032)$, (Table 3$)$.

During operation in group I blood loss ranged between $20-50 \mathrm{ml}$ with a mean amount of blood loss of $33.53 \pm 9.1 \mathrm{ml}$ while in group II the blood loss ranged between $30-55 \mathrm{ml}$ min with a mean of $40.7 \pm 7.1 \mathrm{ml}$ of blood loss and the statistical analysis revealed that there was significant statistical increase in the operative time of group II "sutured group" than in group I "sutureless group" $(\mathrm{P}=0.022)$, (Table 3).

Table 3: Operative time and blood loss during operative procedures in groups of the study. Variable Group I Group II

$\mathbf{P}$




\begin{tabular}{|c|c|c|c|}
\hline $\begin{array}{c}\text { Operative time (Min): } \\
\text { Range } \\
\text { Mean } \pm \text { S.D }\end{array}$ & $\begin{array}{c}40-58 \\
50.3 \pm 4.7\end{array}$ & $\begin{array}{c}49-70 \\
65.5 \pm 5.5\end{array}$ & $\begin{array}{c}\mathbf{0 . 0 3 2} \\
(\mathrm{S})\end{array}$ \\
\hline $\begin{array}{c}\text { Blood loss (ml): } \\
\text { Range } \\
\text { Mean } \pm \text { S.D }\end{array}$ & $\begin{array}{c}20-50 \\
33.5 \pm 9.1\end{array}$ & $\begin{array}{c}30-55 \\
40.7 \pm 7.1\end{array}$ & $\begin{array}{c}\mathbf{0 . 0 2 2} \\
(\mathrm{S})\end{array}$ \\
\hline
\end{tabular}

Twelve patients $(12 / 15,80 \%)$ of group I passed without complications while in group II ten patients $(10 / 15,66.7 \%)$ passed without complications and the statistical analysis revealed that group I "sutureless group" has better outcome than group II "sutured group", $(\mathrm{P}=0.021)$, (Table 4).

Complications encountered in three patients $(3 / 15,20 \%)$, one of them $(1 / 15,6.7 \%)$ was due to superficial wound infections and treated by the use of antibiotic orally; another one $(1 / 15,6.7 \%)$ was due to seroma collection and treated by drainage and antibiotic, and the third one $(1 / 15,6.7 \%)$ occurred late and was due to mesh rejection as a result of infection "deep seated", (Table 4).

In group II, complications encountered in five patients $(5 / 15,33.3 \%)$, one of them $(1 / 15,6.7 \%)$ was due to superficial wound infections and treated by the use of antibiotic orally; two $(2 / 15,13.3 \%)$ was due to seroma collection and treated by drainage and antibiotic, and two $(2 / 15,13.3 \%)$ was due to chronic pain persists post-operatively and treated by postoperative non-steroidal anti-inflammatory drugs (NSAIDs), (Table 4).

Statistical analysis revealed that in spite of increase incidence of complications in group II but there was no significant difference between both groups regarding the complications $(P=0.210)$.

Table 4: Post-operative outcome in patients of the studied groups.

\begin{tabular}{|l|c|c|c|c|c|}
\hline \multirow{2}{*}{ Outcome } & \multicolumn{2}{|c|}{ Group I } & \multicolumn{2}{c|}{ Group II } & \multirow{2}{*}{ P } \\
\cline { 2 - 6 } & No & $\mathbf{\%}$ & No & $\%$ & \\
\hline Pass free & $\mathbf{1 2}$ & $\mathbf{8 0 \%}$ & $\mathbf{1 0}$ & $\mathbf{6 6 . 7 \%}$ & $\mathbf{0 . 0 2 1}^{*}$ \\
\hline Complicated: & $\mathbf{3}$ & $\mathbf{2 0 \%}$ & $\mathbf{5}$ & $\mathbf{3 3 . 3 \%}$ & \\
$\quad$ Superficial wound infection & 1 & $6.7 \%$ & 1 & $6.7 \%$ & \multirow{2}{*}{$\mathbf{0 . 2 1 0}$} \\
Serom & 1 & $6.7 \%$ & 2 & $13.3 \%$ & (NS) \\
Mesh rejection & 1 & $6.7 \%$ & 0 & $0.0 \%$ & \\
Chronic pain & 0 & $0.0 \%$ & 2 & $13.3 \%$ & \\
\hline
\end{tabular}

\section{Discussion}

This study was conducted on 30 cases of fresh inguinal hernia submitted to inguinal hernioplasty "mesh repair" 15 of them were submitted to sutureless mesh "Group I" while the other 15 cases were submitted to sutured mesh "Group II".

Our results revealed that there was no statistical difference between both groups regarding the age. Also, there was no significant difference between both groups regarding gender.
Tarchi and his colleagues, found in their study that the mean age was 62 years and males were more affected (94.3\%) than females $(5.7 \%)$ which run in line with our results ${ }^{(6)}$.

Wang and Zhang in their study found that the age of the affected patients was $48.7 \pm 16.8$ years which was in agreement with our results; while they found that $27 \%$ of their patients were females and $73 \%$ were males which disagrees with our results ${ }^{(5)}$. 
In our study, the type (direct or indirect) and/or the site (right, left or bilateral) of hernia in the groin didn't affect the procedure.

Wang and Zhang in their study found that the right sided inguinal hernia were more common $(67 \%)$ than left sided hernia (33\%), which disagree with our results as we found no predominant type of inguinal hernia ${ }^{(5)}$.

All patients in our study underwent operation by anterior approach using mesh without any intraoperative complications.

Tarchi and his colleagues found in their study that there were no intraoperative complications. Early postoperative complications included hematoma, seroma (5.7\% cases), superficial wound infection (1\%), urinary retention $(0.5 \%)$, and scrotal swelling $(1 \%)$ which was in agreement with our study in both groups ${ }^{(6)}$.

Molegraaf and his colleagues in their study use the self-grip mesh repair in Lechtenstein repair in all of their patients which was in agreement with the results of our study (7).

In spite of increase incidence of complications in sutured group than in sutureless in our study, there was no difference between both groups regarding complications.

Lin and his coworkers in their study found that the incidence of hematomas was significantly higher in the suture fixation group than sutureless group. There was no significant difference between the 2 groups in terms of early recurrence, late chronic pain, late recurrence, wound infection, and mesh infection. The main disadvantage of suture fixation was the duration of operation as the mean operating time was significantly longer than glue fixation and this run in line with our obtained results ${ }^{(\mathbf{8})}$.

Molegraaf and his colleagues concluded in their study that the self-gripping mesh has comparable results with a sutured mesh regarding the incidence of chronic postoperative inguinal pain, recurrence and foreign body sensation. However, long-term results still are based on relatively small patient numbers and outcomes measures are heterogenic. The main advantage of the selfgripping mesh is the consistently significantly reduced operation time which coincides with our results ${ }^{(7)}$.

In addition, Sun and his colleagues, found in their study that self-grip meshes were superior to sutured meshes regarding duration of the operation, hematoma, and recovery time to daily activities. There were no significant differences between the two groups for superficial wound infection, mesh/deep infection, seroma, persisting numbness, postoperative length of stay which was in agreement with the results of our results ${ }^{(3)}$.

Rönkä and his coworkers in their study concluded that the type and size of inguinal hernias were similar in sutureless and sutured groups which were in agreement with our results. They also found that the duration of operation was $32 \pm 9$, and $38 \pm 9$ minutes in the self-gripping, and suture groups, respectively which disagrees with our results. There were no significant differences postoperatively in pain response or need for analgesics, superficial infections, wound seromas between the study groups and this was in agreement with our results ${ }^{(9)}$.

Tarchi and his colleagues found in their study that early postoperative pain was mild and may need non-steroidal anti-inflammatory drug to be given for its relief while chronic postoperative pain with long-term follow up improved even without use of NSAIDs which was in agreement with our study in both groups (6).

\section{Conclusion}

From our study we can conclude that the use of self-grip (sutureless) meshes and sutured meshes in the management of groin hernias have nearly the same results in experienced hands.

\section{Recommendations}

From this study we recommended that: There is still a place for using sutureless technique in management of groin hernia during hernioplasty. Training of our junior staff to do this technique of hernioplasty to have best results and fewer complications that already 
occurred with sutured meshes as chronic pain and infections.

\section{References}

1. Patel HD, Pandya CB and Hathila VP (2014): A Comparative Study between sutureless and Lichtenstein inguinal Mesh hernioplasty. Int. J. Res. Med., 3(3): 109114.

2. Singh RR, Gupta AK, Shah AS et al. (2016): A study about inguinal hernia mesh repairs: plug and patch versus lichtenstein mesh repair technique. Int. Surg. J., 3(4): 1967-1974.

3. Sun P, Cheng X, Deng S et al. (2017): Mesh fixation with glue versus suture for chronic pain and recurrence in Lichtenstein inguinal hernioplasty. Cochr. Database Sys. Rev., 2: 10-114.

4. Sanders DL and Waydia S (2014): A systematic review of randomized control trials assessing mesh fixation in open inguinal hernia repair. Hernia, 18: 165176.
5. Wang $Y$ and Zhang $X$ (2016): Short-term results of open inguinal hernia repair with self-gripping Parietex ProGrip mesh in China: A retrospective study of 90 cases. Asian J. Surg., 39: 218-224.

6. Tarchi P, Cosola D, Germani $P$ et al. (2014): Self-adhesive mesh for Lichtenstein inguinal hernia repair. Experience of a single center. Minerva Chirurgica., 69(3): 167-76.

7. Molegraaft M, Kaufman $R$ and Lange $J$ (2018): Comparison of self-gripping mesh and sutured mesh in open inguinal hernia repair: A meta-analysis of long-term results. Surgery, 163(2): 351-360.

8. Lin H, Zhuang Z, Ma T et al. (2018): A meta analysis of randomized control trials assessing mesh fixation with glue versus suture in Lichtenstein inguinal hernia repair. Medicine, 97: 14-22.

9. Rönkä K, Vironen J, Kossi J et al. (2015): Randomized multi-centertrial comparing glue fixation, self-gripping mesh, and suture fixation of mesh in Lichtenstein hernia repair (Finn Mesh Study). Ann. Surg., 262: 714-720. 